\title{
Study of white matter at the centrum semiovale level with magnetic resonance spectroscopy and diffusion tensor imaging in cerebral small vessel disease
}

\author{
L.A. Huang ${ }^{1}$, X.Y. Ling ${ }^{2}$, C. Li ${ }^{2}$, S.J. Zhang ${ }^{1}$, G.B. Chi ${ }^{2}$ and A.D. Xu' \\ ${ }^{1}$ Department of Neurology, First Affiliated Hospital of Jinan University, \\ Guangzhou, Guangdong, China \\ ${ }^{2}$ Imaging Center, First Affiliated Hospital of Jinan University, Guangzhou, \\ Guangdong, China \\ Corresponding author: A.D. Xu \\ E-mail: andingxu@163.com
}

Genet. Mol. Res. 13 (2): 2683-2690 (2014)

Received March 14, 2013

Accepted October 30, 2013

Published April 8, 2014

DOI http://dx.doi.org/10.4238/2014.April.8.11

\begin{abstract}
White matter lesion (WML) in magnetic resonance imaging is commonly observed in patients with cerebral small vessel disease (SVD), but the pathological mechanism of WML in SVD is still unclear. We observed the metabolism and microscopic anatomy of white matter in SVD patients. Twelve subjects clinically diagnosed with SVD and 6 normal control subjects were examined with magnetic resonance spectroscopy (MRS) and diffusion tensor imaging (DTI). The white matter at the centrum semiovale level was selected as the region of interest (ROI). The ROI metabolism parameters, including N-acetyl-L-aspartic acid (NAA), creatine (Cr), and choline (Cho) were measured by MRS. Microscopic parameters such as mean diffusion (MD) and fractional anisotropy (FA) in ROI were obtained by DTI. Compared with the normal control group, bilateral MD values in the SVD group were significantly elevated, whereas bilateral FA values in
\end{abstract}


SVD were decreased, but the difference was not statistically significant. Additionally, NAA/Cho, Cho/Cr, and NAA/Cr showed no significant statistical differences. Our study suggests that the mechanisms of the SVD cognitive impairment are related to damage of the white matter structures rather than to brain metabolism.

Key words: White matter lesion; Cerebral small vessel disease; Magnetic resonance imaging

\section{INTRODUCTION}

Cerebral small vessel disease (SVD), a subtype of cerebrovascular disease, affects small perforating arteries (Pantoni, 2010; Zhang et al., 2010). Total occlusion of these vessels, which mainly supply basal ganglia and centrum semiovale (CS), causes subcortical lacunar infarction. Additionally, incomplete ischemia leads to leukoaraiosis, which are white matter lesions (WMLs), in conventional magnetic resonance imaging (MRI), with clinical symptoms such as selective cognitive impairment affecting executive function and working memory (Khan et al., 2007; van Dijk et al., 2008).

The principal hypothesis for SVD cognitive impairment involves the interruption of frontal-subcortical circuits, leading to dysexecutive syndromes (Kramer et al., 2002; O'Sullivan et al., 2005; Schmidt et al., 2006). White matter in the CS is the main component of the frontalsubcortical pathway. In addition, WMLs in SVD are often observed in CS. However, whether WMLs and selective cognitive impairment in SVD are correlated is controversial (Roman, 2004; Schmidt et al., 2005; Poggesi et al., 2011). Furthermore, magnetic resonance spectroscopy (MRS) and diffusion tensor imaging (DTI) can detect white matter that is considered normal by conventional MRI with highly sensitive significant metabolic and anatomical abnormalities (O'Sullivan, 2010). However, metabolic and structural studies of white matter in the CS of SVD subjects are rarely reported. We examined cerebral metabolism and the anatomical structure of white matter in the CS to verify the hypothesis of frontal-subcortical pathway damage.

\section{MATERIAL AND METHODS}

\section{Patients}

Similarly to SVD-based studies, the inclusion criteria for SVD subjects were (van Dijk et al., 2008; Nitkunan et al., 2008a,b): 1) MRI indicated subcortical white matter lacunar infarction, with a diameter of less than $15 \mathrm{~mm}$; 2) brain MRI showed subcortical leukoaraiosis; 3) right-handed and the left frontal lobe were the dominant sites; 4) patients provided consent to participate in the study. Exclusion criteria included: 1) patients with comorbidities of cortical or deep nucleus infarction, or focal neurological symptoms and signs including aphasia and paralysis; 2) patients with comorbidities of normal pressure hydrocephalus, brain injury, carbon dioxide poisoning, or hypoglycemia and other diseases which cause cranial MRI white matter abnormalities; 3) confirmed cardiac embolism infarction or intracranial macroangiopathy; 4) patients with comorbidity of depression who could not participate in cognitive examination; 5) patients with poor general conditions and comorbidities with severe diseases of vital organs or history of mental illness. A total of 12 patients, who were admitted 
to our department between October 2009 and December 2010, were included in the SVD, including 7 male and 5 female patients aged between 47 and 82 years. The control group included 6 ( 3 males and 3 females) normal, healthy subjects (age between 52 and 76 years) with no infarction or leukoaraiosis according to cranial MRI and no other major diseases.

Clinical information for SVD subjects and the control group was recorded in detail. Upon approval by the Ethics Committee of the First Affiliated Hospital of Jinan University, all SVDs and control group subjects were provided with free MRS and DTI examination.

\section{MRS and DTI protocols}

Conventional MRI, DTI, and MRS were performed as described previously (Li et al., 2011). The imaging device was a 1.5-T MR scanner (Signa General Electric Medical Systems, Milwaukee, WI, USA) with gradient field magnitude $23 \mathrm{mT} / \mathrm{m}$, gradient switch rate $120 \mathrm{mT} \cdot \mathrm{m}^{-1} \cdot \mathrm{ms}^{-1}$. A head-cervical combined coil was used. Imaging sequences included a T1FLAIR, T2WI, FLAIR, diffusion weighting imaging (DWI), DTI, and MRS. Typical acquisition parameters of conventional MRI were: T1-FLAIR (6 mm thick, 2-mm gap, TR 1475/TE 21.3/TI 750.0 ms) and T2-FLAIR (3 mm thick, gap 0, TR 8000/TE 120/TI2, $200 \mathrm{~ms}$ ). DTI was acquired using a single-shot Echo Planar Imaging (EPI) apparatus with the following parameters: TR 10,000/TE $115 \mathrm{~ms}$; matrix $128 \mathrm{x}$ 128; field of view $24 \times 24 \mathrm{~cm}$; thickness $3 \mathrm{~mm}$, spacing $0 \mathrm{~mm}$; diffusion coefficient $\mathrm{b}=0, \mathrm{~b}=1000$ $\left(\mathrm{s} / \mathrm{mm}^{2}\right)$; gradient orientation of the diffusion sensitivity (Alger, 2010). The scanning time of DTI was $144 \mathrm{~s}$. The scanning baseline was parallel to the antero-posterior commissural line. Axial slices covering the whole brain were obtained using a fast spin echo T2 $(3 \mathrm{~mm}$ thick, gap 0, TR 5660/TE $107.7 \mathrm{~ms}$ ). MRS was performed using the point resolved spectroscopy sequence after suppressing the water peak using a chemical-shift selective excitation with parameters of TR 1000/TE $144 \mathrm{ms,}$ and NEX 1. During image collection, the subject's head was fixed with a pad to avoid motion artifact and shimming was used to reduce anamorphous caused by non-uniform static magnetic field.

Raw data from MRS and DTI were post-processed with the GE AW4.2 software (Signa General Electric Medical Systems). While acquiring the DTI data, the SE-EPI sequence and ramp sampling were considerably reduced without eliminating geometric distortions. Radiography image post-processing was carried by an experienced person who was unaware of the study purpose. The region of interest (ROI) test was the 15 voxel white matters of the CS in each side, and data involved were obtained from the ROI (Figure 1A). Each spectrum was evaluated for the presence of N-acetyl-L-aspartic acid (NAA), creatine (Cr), and choline (Cho). The peak areas of NAA, Cho, and Cr were present in each spectrum for further evaluation (Figure 1B and C). To determine the precise location of the spectroscopic ROI in the anisotropy and diffusivity maps, scanner acquisition was coordinated and the CSI grid was mapped into the DTI space. Notably, after correcting EPI distortions, the threshold values were selected and the mean diffusing (MD) and fractional anisotropy (FA) images were obtained (Figure 2). Additionally, the mean value of MD and FA in the ROI were calculated automatically.

Raw data from MRS and DTI were post-processed with the GE AW4.2 software. While acquiring the DTI data, the SE-EPI sequence and ramp sampling were considerably reduced without eliminating the geometric distortions. An experienced person who was unaware of the study purpose carried out radiograph image post-processing. The ROI examined was the 15 voxels white matter of the CS on each side. The data obtained on each side were from the ROI (Figure 1). Each spectrum was evaluated for the presence of NAA, Cho, and Cr, and 
the peak areas of NAA, Cho, and $\mathrm{Cr}$ were present in each spectrum for further evaluation. To determine the precise location of the spectroscopic ROI in the anisotropy and diffusivity maps, scanner acquisition coordinates and the CSI grid were mapped onto the DTI space. After correction of EPI, distortions and threshold values were selected, and MD and FA images were obtained. The mean value of MD and FA in the ROI were calculated automatically.

\section{Data processing}

Statistical analysis was performed by using SPSS (Version 13.0, SPSS, Inc., Chicago, IL, USA). The Shapiro-Wilk test was conducted for normality, where all variables were normally distributed except for NAA/Cr right. The independent-samples $t$-test and the Wilcoxon rank sum test were conducted to compare SVD and control groups.

\section{RESULTS}

\section{Spectroscopy and tensor results of SVD patients}

Spectroscopy and tensor results of SVD patients and control groups are shown in Tables 1 and 2.

Table 1. Diffusion tensor imaging (DTI) and magnetic resonance imaging (MRS) results of the small vessel disease (SVD) patients in the region of interest (ROI).

\begin{tabular}{lccccccccc}
\hline N/G & MD left & MD right & FA left & FA right & NAA/Cho left NAA/Cho right & NAA/Cr left & NAA/Cr right & Cho/Cr left Cho/Cr right \\
\hline 1/M & 1.190 & 1.180 & 0.216 & 0.193 & 1.150 & 1.168 & 1.764 & 1.670 & 1.533 \\
2/F & 1.050 & 0.985 & 0.407 & 0.445 & 1.610 & 1.800 & 1.900 & 2.050 & 1.180 \\
3/M & 0.847 & 0.863 & 0.52 & 0.465 & 1.398 & 1.502 & 1.879 & 2.009 & 1.344 \\
4/F & 1.060 & 1.070 & 0.392 & 0.371 & 1.068 & 1.229 & 1.557 & 1.794 & 1.457 \\
5/M & 1.080 & 0.954 & 0.220 & 0.227 & 1.130 & 1.010 & 1.880 & 1.580 & 1.660 \\
6/M & 1.050 & 1.130 & 0.44 & 0.383 & 1.137 & 1.195 & 2.003 & 1.860 & 1.760 \\
7/F & 0.943 & 0.879 & 0.416 & 0.482 & 1.740 & 1.800 & 2.000 & 1.96 & 1.090 \\
8/F & 0.924 & 0.886 & 0.440 & 0.422 & 1.766 & 1.92 & 2.047 & 1.828 & 1.158 \\
9/M & 1.030 & 1.210 & 0.450 & 0.384 & 1.391 & 1.602 & 2.318 & 2.316 & 1.666 \\
10/M & 1.240 & 1.260 & 0.399 & 0.343 & 1.156 & 1.080 & 2.051 & 1.932 & 1.773 \\
11/F & 0.761 & 0.882 & 0.530 & 0.441 & 1.328 & 1.422 & 1.809 & 2.266 & 1.453 \\
12/M & 0.690 & 0.889 & 0.306 & 0.269 & 1.250 & 1.500 & 1.750 & 1.930 & 1.393 \\
\hline
\end{tabular}

$\mathrm{N}=$ number of cases; $\mathrm{G}=$ gender; $\mathrm{M}=$ male; $\mathrm{F}=$ female; $\mathrm{MD}=$ mean diffusions; $\mathrm{FA}=$ fractional anisotropy; $\mathrm{NAA}=\mathrm{N}$-acetyl-L-aspartic acid $; \mathrm{Cho}=$ choline $; \mathrm{Cr}=$ creatine

Table 2. DTI and MRS results in the ROI of the control group.

\begin{tabular}{lccccccccc}
\hline N/G & MD left & MD right & FA left & FA right & NAA/Cho left NAA/Cho right & NAA/Cr left & NAA/Cr right & Cho/Cr left & Cho/Cr right \\
\hline 1/M & 0.696 & 0.860 & 0.451 & 0.366 & 1.140 & 1.310 & 1.480 & 1.710 & 1.300 \\
2/F & 0.781 & 0.880 & 0.523 & 0.449 & 1.699 & 1.711 & 2.236 & 2.640 & 1.320 \\
3/F & 0.799 & 0.863 & 0.373 & 0.320 & 1.720 & 1.540 & 2.740 & 1.990 & 1.600 \\
4/F & 0.801 & 0.839 & 0.447 & 0.443 & 1.420 & 1.410 & 1.820 & 1.820 & 1.280 \\
5/M & 0.756 & 0.943 & 0.484 & 0.402 & 1.340 & 1.320 & 2.100 & 1.850 & 1.560 \\
6/M & 0.597 & 0.963 & 0.598 & 0.382 & 1.320 & 1.310 & 1.980 & 1.870 & 1.510 \\
\hline
\end{tabular}

For abbreviations, see Table 1. 


\section{MRS and DTI images of the SVD Case 1}

The white matter in the ROI was divided into 15 voxels by average (Figure 1A). Data were obtained from 15 voxels in the ROI on each side and the peak areas of NAA, Cho, and $\mathrm{Cr}$ were identified in each spectrum (Figure 1B). Additionally, the mean values of NAA, Cho, and $\mathrm{Cr}$ and metabolite ratios in the ROI were calculated automatically (Figure 1C).

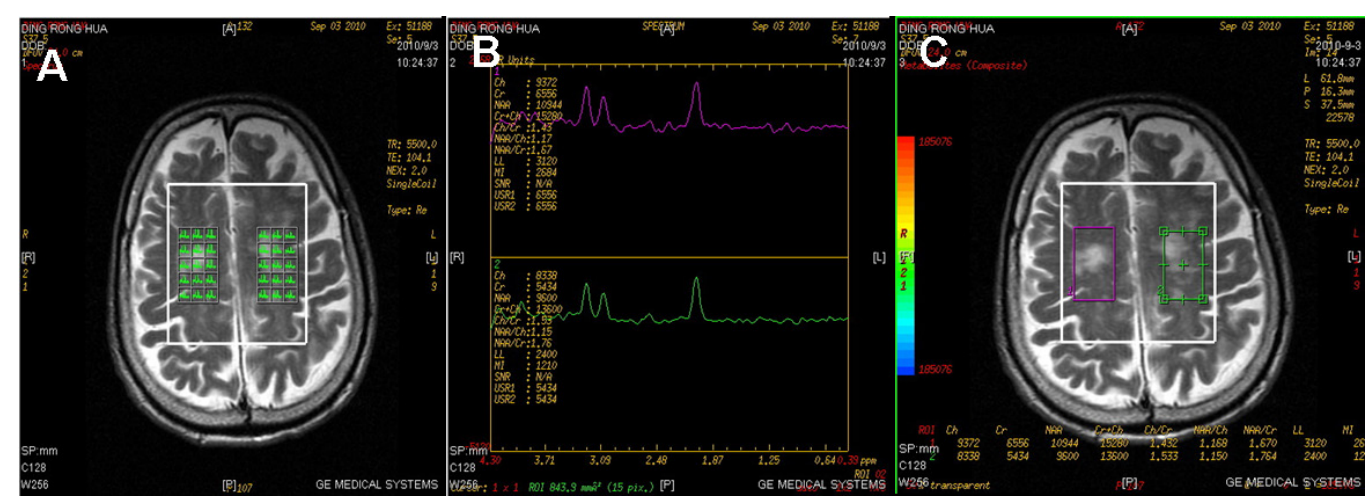

Figure 1. MRS images of the SVD Case 1. The white matter in the ROI was divided into 15 voxels by average (A). Data were obtained from 15 voxels in the ROI on each side and the peak areas of NAA, Cho, and Cr were found in each spectrum (B). Also, the mean values of NAA, Cho, and $\mathrm{Cr}$ and metabolite ratios in the ROI were calculated automatically $(\mathbf{C})$. For abbreviations, see Table 1.

Notably, the image was obtained and mean FA value of the ROI was calculated automatically (Figure 2A), and the MD image was obtained and mean MD value of the ROI was calculated automatically (Figure 2B).

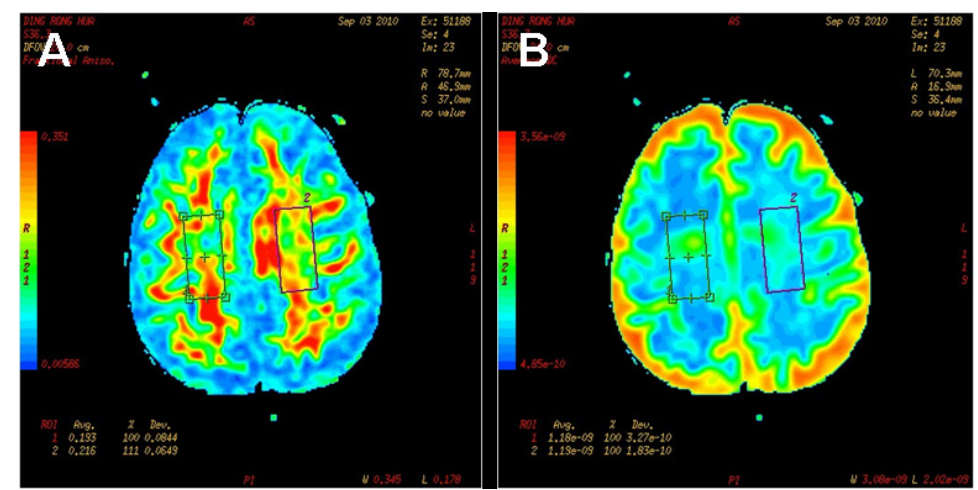

Figure 2. DTI images of the SVD Case 1. It is to be noted that image was obtained and mean FA value of the ROI was calculated automatically (A), and MD image was obtained and mean MD value of the ROI was calculated automatically (B). For abbreviations, see Table 1.

\section{FA and MRS parameters in the ROI}

By comparing the FA and MRS parameters in the ROI, we found no significant differ- 
ences in the MRS parameters between the 2 groups (Table 3 ). However, there was a statistical difference in the either side MD values $(\mathrm{P}<0.05)$ between SVD and control. We also found that either side FA values in SVD were lower than those in the control group, although the difference was not statistically significant (Table 4).

Table 3. Comparing MRS parameters in SVD (means \pm SD).
\begin{tabular}{lcccc}
\hline MRS & SVD & Control & $t$-test value & P \\
\hline NAA/Cho left & $1.344 \pm 0.245$ & $1.440 \pm 0.228$ & 0.803 & 0.434 \\
NAA/Cho right & $1.436 \pm 0.304$ & $1.434 \pm 0.163$ & 1.025 & 0.985 \\
NAA/Cr left & $1.913 \pm 0.192$ & $2.059 \pm 0.423$ & 0.187 & 0.320 \\
NAA/Cr right & $1.933 \pm 0.215$ & $1.980 \pm 0.336$ & 0.185 & $0.892 *$ \\
Cho/Cr left & $1.448 \pm 0.235$ & $1.428 \pm 0.144$ & 0.144 & 0.856 \\
Cho/Cr right & $1.391 \pm 0.233$ & $1.380 \pm 0.101$ & 0.887 \\
\hline
\end{tabular}

*Wilcoxon rank sum test, $\mathrm{Z}=0.187$. For abbreviations, see Table 1 .

Table 4. Comparing DTI parameters in SVD (means \pm SD).

\begin{tabular}{lcccc}
\hline DTI & SVD & Control & $t$-test value & P \\
\hline MD left & $0.989 \pm 0.163$ & $0.738 \pm 0.079$ & 3.513 & 0.003 \\
MD right & $1.016 \pm 0.147$ & $0.891 \pm 0.050$ & 2.640 & 0.019 \\
FA left & $0.395 \pm 0.101$ & $0.479 \pm 0.076$ & 1.802 & 0.090 \\
FA right & $0.369 \pm 0.094$ & $0.394 \pm 0.049$ & 0.601 & 0.556 \\
\hline
\end{tabular}

For abbreviations, see Table 1.

\section{DISCUSSION}

MRS can non-invasively measure cerebral metabolism, including NAA, Cho, and Cr. The level of NAA, which is primarily located in mature neurons and neurites such as axons, indicates the functional status of the neuron. Involved in the formation of cellular membrane as a precursor of acetylcholine (neurotransmitter), Cho indicates the total choline cerebral content. Imaging parameters obtained from MD and FA are used to quantitatively detect abnormalities in the microstructure of the cerebral white matter. These white matter tracts in the cerebral white matter are highly directional. While MD indicates the speed of diffusion but not its anisotropism, i.e., the mean magnitude of the diffusion of water molecules in all directions, FA reflects anisotropism characteristics of water molecules in the brain (Oouchi et al., 2007; Alger, 2010).

Regarding information of region, rather than the whole brain, differences in white matter integration is valuable, particularly when the goal is to correlate white matter measures with cognitive measures (Nitkunan et al., 2008a; Patel and Markus, 2011). White matters in the CS has been suggested as a key region for detecting the white matter damaged by various cognition impairments such as vascular dementia, extremely preterm infants, and norexia nervosa, among others (Skiöld et al., 2010; Zhang et al., 2011; Blasel et al., 2012).

Previous studies have shown that WMLs affect the clinical outcome in patients with acute striatocapsular stroke, and WMLs were presumed to disrupt frontal-subcortical network integration (Held et al., 2012). Using DTI technology and dynamic observation, Charlton et al. (2010) studied 84 healthy middle-aged and elderly subjects and found that changes in WMLs and cerebral volume were not correlated with cognition, while according to DTI the values of FA and MD were significantly correlated with the working memory during cognitive decline. 
In our study, MD is more significant than the FA between SVD and control groups. Because the value of MD indicates the speed of diffusion and is relatively independent of the location measured in the brain, FA values are very dependent on the degree of white matter organization and finally in the location where it is measured (Stebbins et al., 2007; van Norden et al., 2012). Because multiple fibers are found in the ROI, they may all have different destinations. FA in ROI does not necessarily reflect an underlying lower structural integration. Our study further highlighted that compared to the control groups, frontal-subcortical circuit anatomical integration are damaged in SVD.

Previously, MRS research studies of 20 healthy elderly individuals found that executable and noticeable abilities were correlated with NAA concentration in the frontal white matter (Valenzuela et al., 2000). Nitkunan A et al. (2009) studied SVD and control subjects in the entire central region of CS with MRS and DTI, and found that mean DTI parameters were changed in SVD and correlated mean NAA. However, the ROI in Nitkunan studies were not accurately selected. Not only the entire central region of CS, but also other structures such as the callosum, consists of white matter. Beppu et al. (2012) examined bilateral white matter separately in the CS with DTI in carbon monoxide, which could poison the patient, and found that the mean FA was significantly lower in patients displaying chronic symptoms than in patients without chronic symptoms or controls. The ROI selected in our study was similar to that used in Beppu's study, as bilateral white matter selected as ROI is widely used in many MRS studies (Skoch et al., 2008; Alger, 2010). Quantitative study of MRS more accurately measures the concentration of metabolites such as NAA, Cho, and Cr, as well as the relationship of their rates with each other, such as NAA/Cho, NAA/Cr and Cho/Cr. The metabolite ratio of quantitative MRS is widely used in cerebral disease to eliminate the influence of any external magnetic field or living organisms (Skoch et al., 2008; Nitkunan et al., 2009). By selecting each side white matter in the CS as ROI and calculating the metabolic ratio, our study found no significant statistical difference in MRS parameters, including NAA/Cho, NAA/Cr and Cho/Cr, between the SVD and control groups, suggesting that cerebral metabolism may not have a major role in pathogenesis of SVD. Nitkunan A et al. (2009) found that differences in NAA between SVD patients and controls were not significant after controlling for T2 lesion volume within each ROI, and no correlations between cognitive scores and any brain metabolite in SVD patients were observed.

In conclusion, our results showed that SVD subjects had anatomical damage but no cerebral metabolism changes in centrum semiovale white matter, suggesting that the mechanisms of SVD cognitive impairment are related to damage to white matter structures, but not to brain metabolism. The loss of white matter integration in SVD identified by DTI is not likely caused by cerebral metabolism changes, validating large-sample and multi-center combination studies.

\section{ACKNOWLEDGMENTS}

Research supported by the Fund of Province Science and Technology of Guangdong China (\#2011B050300012), and the National Natural Science Foundation China (\#81271280).

\section{REFERENCES}

Alger JR (2010). Quantitative proton magnetic resonance spectroscopy and spectroscopic imaging of the brain: a didactic review. Top. Magn. Reson. Imaging 21: 115-128.

Beppu T, Fujiwara S, Nishimoto H, Koeda A, et al. (2012). Fractional anisotropy in the centrum semiovale as a quantitative indicator of cerebral white matter damage in the subacute phase in patients with carbon monoxide poisoning: 
correlation with the concentration of myelin basic protein in cerebrospinal fluid. J. Neurol. 259: 1698-1705.

Blasel S, Pilatus U, Magerkurth J, von Stauffenberg M, et al. (2012). Metabolic gray matter changes of adolescents with anorexia nervosa in combined MR proton and phosphorus spectroscopy. Neuroradiology 54: 753-764.

Charlton RA, Schiavone F, Barrick TR, Morris RG, et al. (2010). Diffusion tensor imaging detects age related white matter change over a 2 year follow-up which is associated with working memory decline. J. Neurol. Neurosurg. Psychiatry 81: 13-19.

Held V, Szabo K, Bazner H and Hennerici MG (2012). Chronic small vessel disease affects clinical outcome in patients with acute striatocapsular stroke. Cerebrovasc. Dis. 33: 86-91.

Khan U, Porteous L, Hassan A and Markus HS (2007). Risk factor profile of cerebral small vessel disease and its subtypes. J. Neurol. Neurosurg. Psychiatry 78: 702-706.

Kramer JH, Reed BR, Mungas D, Weiner MW, et al. (2002). Executive dysfunction in subcortical ischaemic vascular disease. J. Neurol. Neurosurg. Psychiatry 72: 217-220.

Li C, Ling X, Liu S, Xu A, et al. (2011). Early detection of secondary damage in ipsilateral thalamus after acute infarction at unilateral corona radiata by diffusion tensor imaging and magnetic resonance spectroscopy. BMC Neurol. 11: 49.

Nitkunan A, Charlton RA, McIntyre DJ, Barrick TR, et al. (2008a). Diffusion tensor imaging and MR spectroscopy in hypertension and presumed cerebral small vessel disease. Magn. Reson. Med. 59: 528-534.

Nitkunan A, Barrick TR, Charlton RA, Clark CA, et al. (2008b). Multimodal MRI in cerebral small vessel disease: its relationship with cognition and sensitivity to change over time. Stroke 39: 1999-2005.

Nitkunan A, Charlton RA, Barrick TR, McIntyre DJ, et al. (2009). Reduced N-acetylaspartate is consistent with axonal dysfunction in cerebral small vessel disease. NMR Biomed. 22: 285-291.

O'Sullivan M (2010). Imaging small vessel disease: lesion topography, networks, and cognitive deficits investigated with MRI. Stroke 41: S154-S158.

O'Sullivan M, Barrick TR, Morris RG, Clark CA, et al. (2005). Damage within a network of white matter regions underlies executive dysfunction in CADASIL. Neurology 65: 1584-1590.

Oouchi H, Yamada K, Sakai K, Kizu O, et al. (2007). Diffusion anisotropy measurement of brain white matter is affected by voxel size: underestimation occurs in areas with crossing fibers. Am. J. Neuroradiol. 28: 1102-1106.

Pantoni L (2010). Cerebral small vessel disease: from pathogenesis and clinical characteristics to therapeutic challenges. Lancet Neurol. 9: 689-701.

Patel B and Markus HS (2011). Magnetic resonance imaging in cerebral small vessel disease and its use as a surrogate disease marker. Int. J. Stroke 6: 47-59.

Poggesi A, Pantoni L, Inzitari D, Fazekas F, et al. (2011). 2001-2011: A decade of the LADIS (Leukoaraiosis and DISability) study: what have we learned about white matter changes and small-vessel disease? Cerebrovasc. Dis. 32: $577-588$.

Roman GC (2004). Age-associated white matter lesions and dementia: are these lesions causal or casual? Arch. Neurol. 61: 1503-1504.

Schmidt R, Ropele S, Enzinger C, Petrovic K, et al. (2005). White matter lesion progression, brain atrophy, and cognitive decline: the Austrian stroke prevention study. Ann. Neurol. 58: 610-616.

Schmidt R, Enzinger C, Ropele S, Schmidt H, et al. (2006). Subcortical vascular cognitive impairment: similarities and differences with multiple sclerosis. J. Neurol. Sci. 245: 3-7.

Skiöld B, Horsch S, Hallberg B, Engstrom M, et al. (2010). White matter changes in extremely preterm infants, a population-based diffusion tensor imaging study. Acta Paediatr. 99: 842-849.

Skoch A, Jiru F and Bunke J (2008). Spectroscopic imaging: basic principles. Eur. J. Radiol. 67: 230-239.

Stebbins GT, Smith CA, Bartt RE, Kessler HA, et al. (2007). HIV-associated alterations in normal-appearing white matter: a voxel-wise diffusion tensor imaging study. J. Acquir. Immune. Defic. Syndr. 46: 564-573.

Valenzuela MJ, Sachdev PS, Wen W, Shnier R, et al. (2000). Dual voxel proton magnetic resonance spectroscopy in the healthy elderly: subcortical-frontal axonal $\mathrm{N}$-acetylaspartate levels are correlated with fluid cognitive abilities independent of structural brain changes. Neuroimage 12: 747-756.

van Dijk EJ, Prins ND, Vrooman HA, Hofman A, et al. (2008). Progression of cerebral small vessel disease in relation to risk factors and cognitive consequences: Rotterdam Scan study. Stroke 39: 2712-2719.

van Norden AG, de Laat KF, van Dijk EJ, van Uden IW, et al. (2012). Diffusion tensor imaging and cognition in cerebral small vessel disease: the RUN DMC study. Biochim. Biophys. Acta 1822: 401-407.

Zhang AJ, Yu XJ and Wang M (2010). The clinical manifestations and pathophysiology of cerebral small vessel disease. Neurosci. Bull. 26: 257-264.

Zhang B, Wen CY, Wang L and Zhang X (2011). Functional MRI and cognition assessment in subcortical ischemic vascular disease. Zhonghua Nei Ke. Za Zhi. 50: 411-415. 